\title{
Outcomes of surgical and transcatheter aortic valve replacement in the octogenarians-surgery still the gold standard?
}

\author{
Sameer A. Hirji ${ }^{1 *}$, Fernando Ramirez-Del Val ${ }^{1 *}$, Ahmed A. Kolkailah ${ }^{1}$, Julius I. Ejiofor ${ }^{1}$, Siobhan \\ McGurk $^{1}$, Ritam Chowdhury ${ }^{2}$, Jiyae Lee ${ }^{1}$, Pinak B. Shah ${ }^{3}$, Piotr S. Sobieszczyk ${ }^{3}$, Sary F. Aranki ${ }^{1}$, Marc P. \\ Pelletier ${ }^{1}$, Prem S. Shekar ${ }^{1}$, Tsuyoshi Kaneko ${ }^{1}$ \\ ${ }^{1}$ Division of Cardiac Surgery, ${ }^{2}$ Center for Surgery and Public Health (CSPH), ${ }^{3}$ Department of Cardiovascular Medicine, Brigham and Women's \\ Hospital, Harvard Medical School, Boston, MA, USA \\ *These authors contributed equally to this work. \\ Correspondence to: Tsuyoshi Kaneko, MD. Division of Cardiac Surgery, Brigham and Women's Hospital, 75 Francis St, Boston, Ma 02115, USA. \\ Email: tkaneko2@partners.org.
}

Background: Contemporary options for aortic valve replacement (AVR) include transcatheter and surgical approaches (TAVR and SAVR). As evidence accrues for TAVR in high and intermediate risk patients, some clinicians advocate that all patients aged over 80 years should only receive TAVR. Our aim was to investigate the utility of SAVR and minimally invasive AVR (mAVR) among octogenarians in the current era of TAVR.

Methods: From 2002 to 2015, 1,028 octogenarians underwent isolated AVR; 306 TAVR and 722 SAVR, of which 378 patients underwent mAVR. Logistic regression and Cox modeling were used to evaluate overall operative mortality and mid-term survival, respectively. Patients were stratified based on procedural approaches (mAVR or full sternotomy for SAVR, and transfemoral or alternate access for TAVR). Median follow-up was 35 [interquartile range (IQR) 14-65] months.

Results: Compared to SAVR patients, TAVR patients were relatively older (86.2 versus 84.2 years) with co-morbidities such as chronic kidney disease (CKD), diabetes mellitus (DM), cerebrovascular disease (CVD), and prior myocardial infarction $(\mathrm{MI})$, all $\mathrm{P}<0.05$. The mean STS-PROM for the TAVR group was statistically higher, 6.81 versus 5.58 for the SAVR group $(\mathrm{P}<0.001)$. The median in-hospital LOS was statistically higher for the SAVR group $(\mathrm{P}<0.05)$. Cox proportional hazard modeling, adjusted for temporal differences in procedure and patient selection, identified age, New York Heart Association (NYHA) class III/IV, preoperative creatinine, severe chronic lung disease, prior cardiac surgery as significant predictors of decreased survival (all $\mathrm{P}<0.05$ ), while type of intervention (approach) was non-contributory. Adjusted operative mortality stratified by procedure approaches was similar between full sternotomy SAVR and mAVR, and between alternative access and transfemoral TAVR.

Conclusions: After adjusting for confounders, TAVR (regardless of approach), SAVR, and mAVR had comparable operative mortality and mid-term survival. Treatment decisions should be individualized with consensus from a multi-disciplinary heart team, taking into account patient co morbidities, frailty, and quality of life. We believe certain patient groups will still benefit from SAVR even in this elderly population.

Keywords: Aortic valve replacement (AVR); octogenarians; transcatheter

Submitted Jul 23, 2017. Accepted for publication Aug 03, 2017.

doi: 10.21037/acs.2017.08.01

View this article at: http://dx.doi.org/10.21037/acs.2017.08.01 


\section{Introduction}

Aortic stenosis is the most common native valve disease, reportedly affecting up to $5 \%$ of the elderly population, with increasing incidences over time as life expectancy increases $(1,2)$. Traditionally, surgical aortic valve replacement (SAVR) has demonstrated improved survival and definitive treatment in symptomatic patients $(3,4)$. However, contemporary options for aortic valve replacement (AVR) include transcatheter AVR (TAVR), which has evolved as a suitable alternative to SAVR.

Within the last decade especially, significant evidence has emerged demonstrating the efficacy and safety of TAVR in high and intermediate risk patients using various validated, randomized clinical trials such as the Placement of Aortic Transcatheter Valve (PARTNER) IA, IB and recently IIA trials (5-7). These results are promising and, in many ways, have begun to shift the paradigm in management of aortic disease among octogenarians and nonagenarians. In fact, some clinicians believe that all patients aged over 80 years should receive TAVR given their comorbidities and overall frailty.

Unfortunately, TAVR approaches are limited to certain high-volume or academic centers with transcatheter capabilities. These approaches are also associated with steep learning curves for trainees and higher patient costs, with limited follow-up data on valve performance and duration long term. Keeping these concerns in mind, our aim was to compare the outcomes of TAVR and SAVR, including minimally invasive AVR (mAVR) in the elderly and assess whether SAVR still remains a viable and perhaps the goldstandard approach for management among octogenarians in the current era of TAVR.

\section{Methods}

\section{Data source}

Following approval by the institutional review board, we identified all octogenarians who underwent isolated AVR procedures at our institution between January 2002 and December 2015. Patients undergoing concurrent coronary artery bypass grafting (CABG) procedure, those undergoing replacement of mitral or tricuspid valves, and those undergoing any repeat valvular replacement or repair procedure were excluded from the analysis.

\section{Demographic data}

Patient demographic, operative and in-hospital outcomes were extracted from our hospital's electronic medical records, and data for TAVR patients was derived from our institutions' Society of Thoracic Surgeons (STS)/ American College of Cardiology (ACC) Transcatheter Valve Therapy (TVT) Registry data file. All variables presented were coded according to specifications from the Adult Cardiac Surgery Database version 2.52 specifications, unless otherwise indicated. Chronic kidney disease (CKD) was defined a priori as a preoperative creatinine $\geq 2.0 \mathrm{mg} / \mathrm{dL}$ or most recent clinical documentation of renal disease. Echocardiographic data was also utilized to obtain pertinent preoperative and postoperative aortic valve hemodynamic data. Pre-procedure echocardiographic data was extracted from the most recent pre-operative study when available, or the intra-operative trans-esophageal echocardiography (TEE). The degree of post-procedure AR was derived from operative notes or echocardiographic studies.

\section{Surgical approach}

At our institution, the most commonly utilized mAVR approach is via the hemi-sternotomy approach, a technique we have previously described and have shown comparable outcomes for, compared to the conventional full sternotomy approach $(8,9)$. Briefly, the upper-hemi-sternotomy at the level of the 4th intercostal space is utilized. For venous drainage, either the right atrium or right femoral vein is cannulated (9). Another mAVR approach via the rightsided thoracotomy is also utilized; a total of 50 patients underwent $\mathrm{mAVR}$ via this technique.

\section{Patient selection}

The selection of mAVR versus full AVR was at the surgeon's discretion. Patients selected for TAVR were based on the FDA approved criteria. Patients who were deemed high or prohibitive surgical risk by the heart team were treated with TAVR. High risk was defined as STSpredicted risk of mortality (PROM) $\geq 8.0$ or other criteria such as patient frailty, cirrhosis, or pulmonary hypertension, that were not captured by the STS score.

\section{Outcome of interest}

Primary outcomes of interest were operative mortality and post-operative survival. Secondary outcomes included length of stay (LOS), permanent stroke, and postoperative resolution of aortic regurgitation (AR). We defined 
operative mortality as any death occurring in-hospital during the index admission or within 30 days of surgery, if discharged. The STS-PROM score was calculated using the 2008 algorithm. The observed-to-expected ratio (O/E ratio) was the percent operative mortality divided by the STSPROM.

We further stratified each procedure into various subgroups, for additional analysis, based on procedure approaches. For instance, TAVR was further stratified according to the transfemoral approach (TAVT-TF) or alternate access via either subclavian or transaortic approaches (TAVR-Alt). Similarly, SAVR was divided into the full sternotomy group (SAVR) versus the mAVR. Survival data was obtained from our internal research data repository, routine patient or clinic follow-up, query of the social security disability insurance (SSDI), and our state's Department of Public Health Records. Follow-up time was calculated in months from the date of surgery to the date of death or June 30, 2016 (i.e., 6 months after the study period). There was $99 \%$ follow-up for patient survival. For patients lost to follow-up, observation time was censored at the point of last known clinical contact.

\section{Statistical analysis}

Normally distributed variables were expressed as a mean with standard deviation, and compared using Student's $t$-test with Levene's test for homogeneity for variance. Nonnormally distributed variables were expressed as a median with interquartile range (IQR), and were compared using Mann-Whitney $U$ tests. On the other hand, categorical variables were presented as number and percentages, and were compared using $\chi^{2}$ or Fisher's exact tests. All analyses were conducted using IBM SPSS Statistics version 23.0 (IBM Corporation, Armonk, NY, USA) with $\mathrm{P} \leq 0.05$ as the criterion for significance.

Unadjusted survival was evaluated by Kaplan-Meier analysis with log-rank tests. Adjusted survival was evaluated using forward stepwise Cox proportional hazard modeling. Variables selected included those found to be significantly different between groups on univariate analyses, variables known to be contributors to all-cause mortality, and those deemed clinically meaningful to the procedure subgroups. Continuous variables included for analyses were age (years), ejection fraction (\%), and preoperative creatinine $(\mathrm{mg} / \mathrm{dL})$. Categorical variables evaluated included congestive heart failure (CHF), New York Heart Association (NYHA) class III/IV, CKD, re-operative surgery, diabetes mellitus
(DM), hypertension, cerebrovascular accident (CVA), cerebrovascular disease (CVD), peripheral vascular disease (PVD), emergent operative status, and type of procedure. Transcatheter procedures were not available outside of clinical trials prior to 2012, and our institutional practice for referral for TAVR versus SAVR were not analogous. To address confounding due to the practice and temporal differences, the year of surgery was entered in our model.

\section{Results}

\section{Patient demographics}

Our overall cohort consisted of 1,028 patients, including 306 TAVR patients and 722 SAVR patients. Of the SAVR group, 378 patients underwent mAVR, 328 via hemisternotomy and 50 via right sided-thoracotomy. The mean patient age was $84.4 \pm 3.4$ (range $80-94$ for SAVR and 80-97 for TAVR) years and 52.3\% [538] were women. Preoperatively, $7.5 \%$ (77 patients) had CKD with a mean creatinine of $1.2 \pm 0.7 \mathrm{mg} / \mathrm{dL}$. Most patients, i.e., $79.7 \%$ (819 patients) had hypertension, while the prevalence of diabetes, CVD, and previous MI was 23.9\% (246 patients), $7.8 \%$ (80 patients), and $13.5 \%$ (139 patients), respectively. Notably, $63.5 \%$ (653 patients) had symptoms of CHF in the 2 weeks before surgery, and 59.4\% (611 patients) were in the NYHA class III or IV classification. Overall, the mean STS-PROM score was $5.96 \pm 3.87$, with a median follow-up of 35 months (IQR 14-65) as shown in Table 1.

\section{TAVR and SAVR subgroups}

Compared to SAVR patients, TAVR patients were older (86.2 vs. 84.1 years) and had a higher rate of CKD (13.1\% vs. $5.1 \%), \mathrm{DM}(35.0 \%$ vs. $19.3 \%)$, CVD (12.7\% vs. $5.7 \%)$, and prior $\mathrm{MI}(24.2 \%$ vs. $9.0 \%)$, all $\mathrm{P} \leq 0.001$. The mean STS-PROM for the TAVR group was statistically higher, $6.81 \pm 4.54$ versus $5.58 \pm 3.48$ for the SAVR group $(\mathrm{P} \leq 0.001)$. Additionally, TAVR patients tended to be much sicker as reflected by their CHF history $(83.3 \%$ vs. $55.1 \%)$ and overall NYHA class (i.e., III/IV) (79.1\% vs. $51.1 \%)$, all $\mathrm{P} \leq 0.001$. Both these groups, however, were similar in terms of gender composition, hypertension history, and emergent status of the procedure (Table 1).

\section{Operative characteristics}

Table 2 shows operative and in-hospital outcomes. The mean 


\begin{tabular}{|c|c|c|c|c|}
\hline Age (years), mean \pm SD & $84.4 \pm 3.4$ & $84.1 \pm 3.2$ & $86.2 \pm 3.9$ & $0.001^{*}$ \\
\hline Women, n (\%) & $538(52.3)$ & $377(52.2)$ & $161(52.6)$ & 1.000 \\
\hline Renal failure, n (\%) & $77(7.5)$ & $37(5.1)$ & $40(13.1)$ & $0.001^{*}$ \\
\hline Diabetes, n (\%) & $246(23.9)$ & 139 (19.3) & $107(35.0)$ & $0.001^{*}$ \\
\hline Cerebrovascular disease, n (\%) & $80(7.8)$ & $41(5.7)$ & $39(12.7)$ & $0.001^{*}$ \\
\hline HTN, n (\%) & $819(79.7)$ & $574(79.5)$ & $245(80.1)$ & 1.000 \\
\hline Previous MI, n (\%) & $139(13.5)$ & $65(9.0)$ & $74(24.2)$ & $0.001^{*}$ \\
\hline Emergent/salvage status, n (\%) & $7(0.7)$ & $5(0.7)$ & $2(0.7)$ & 1.000 \\
\hline Previous CABG or valve surgery, $\mathrm{n}(\%)$ & $231(22.5)$ & $134(18.6)$ & $97(31.7)$ & 0.052 \\
\hline STS-PROM mean \pm SD & $5.96 \pm 3.87$ & $5.58 \pm 3.48$ & $6.81 \pm 4.54$ & $0.001^{*}$ \\
\hline Follow-up time (months) median (IQR) & $35.0[14-65]$ & $52.0[26-78]$ & 14.0 [8-23] & - \\
\hline
\end{tabular}

preoperative aortic valve gradient was $45.5 \pm 16.3 \mathrm{mmHg}$ in the overall cohort, but statistically higher in the SAVR group versus the TAVR group $(48.2 \pm 17.4$ vs. $43.7 \pm 12.8$, respectively), all $\mathrm{P} \leq 0.001$. The TAVR group had more patients who had undergone a previous AVR procedure $(12.1 \%$ vs. $2.9 \%)$ and were less likely to have had cardiopulmonary bypass $(23.9 \%$ vs. $100 \%, \mathrm{P} \leq 0.001)$ compared to SAVR group. In terms of devices implanted, $92 \%$ of SAVR patients received bioprosthetic valves and $8 \%$ had mechanical valves. On the other hand, the TAVR valves implanted included Sapien (28.4\%), Sapien XT (23.9\%), Sapien 3 (30.7\%) and CoreValve (15\%) (Table 2).

\section{In-hospital outcomes}

Operative mortality was similar between the two groups (4.2\% in TAVR vs. $5.1 \%$ in SAVR, $\mathrm{P}=0.64$ ), as was the incidence of stroke (5.6\% in TAVR vs. $6.4 \%, \mathrm{P}=0.47)$. None of the patients in the SAVR group had early postoperative evidence of residual AR, unlike the TAVR group, where the incidence of postoperative mild and moderate residual AR (para-valvular leaks) was $6.9 \%$ and $2.0 \%$, respectively.
Similarly, the TAVR group had no patients with new onset renal failure, compared to $3.7 \%$ (27 patients) in the SAVR group (Table 2). The median intensive care unit (ICU) stay and in-hospital LOS was significantly longer for the SAVR group [51 (IQR 28-96) h versus 6 (IQR 0-28) h, and 8 (IQR 6-11) days versus 3 (IQR 2-6) days, all $\mathrm{P} \leq 0.001$ vs. TAVR, respectively).

\section{Mid-term survival}

There were $24(2.3 \%)$ patients lost to survival followup. Median follow-up time was 52 months for SAVR patients and 14 months for TAVR, for a total of 3,719 patient-years of follow-up, during which there were 369 deaths. The Kaplan-Meier analysis (Figure 1A) shows that SAVR patients had significantly better long-term survival compared to TAVR patients; unadjusted survival at $12,24,36$, and 48 months was $86 \%, 82 \%, 78 \%$ and $73 \%$ respectively for SAVR versus $83 \%, 75 \%, 64 \%$, and $52 \%$ for TAVR $(\mathrm{P}=0.024)$. Figure $1 B$ shows that adjusted survival did not differ significantly across groups. Using Cox proportional hazard modeling with year of surgery 


\begin{tabular}{|c|c|c|c|c|}
\hline Preop valve gradient, mean \pm SD & $45.5 \pm 16.3$ & $48.2 \pm 17.4$ & $43.7 \pm 12.8$ & $0.001^{*}$ \\
\hline Previous AVR, n (\%) & $58(5.6)$ & $21(2.9)$ & $37(12.1)$ & $0.001^{*}$ \\
\hline Required CPB, n (\%) & 795 (77.3) & $722(100.0)$ & $73(23.9)$ & $0.001^{*}$ \\
\hline Cross clamp time (min), median (IQR) & $69.0[56-85]$ & $69.0[56-85]$ & $62.0[14-62]$ & 0.850 \\
\hline \multicolumn{5}{|l|}{ Implant device type, n (\%) } \\
\hline Bioprosthetic & - & $667(92.4)$ & - & - \\
\hline Mechanical & - & $55(7.6)$ & - & - \\
\hline Sapien 3 & - & - & $94(30.7)$ & - \\
\hline CoreValve & - & - & $46(15.0)$ & - \\
\hline \multicolumn{5}{|l|}{ Postoperative complications } \\
\hline \multicolumn{5}{|l|}{ Residual AR } \\
\hline None, n (\%) & $970(94.4)$ & $722(100.0)$ & $248(81.0)$ & - \\
\hline Trace, $\mathrm{n}(\%)$ & $32(3.1)$ & & $32(10.5)$ & - \\
\hline Mild, $\mathrm{n}(\%)$ & $21(2.0)$ & & $21(6.9)$ & - \\
\hline Moderate, n (\%) & $6(0.6)$ & & $6(2.0)$ & - \\
\hline Observed/expected ratio & 0.73 & 0.95 & 0.62 & - \\
\hline
\end{tabular}

*, P<0.05 was statistically significant. AR, aortic regurgitation; ICU, intensive care unit; AVR, aortic valve repair; CPB, cardiopulmonary bypass; LOS, length of stay.

to adjust for the temporal differences in procedure availability, and in patient selection and clinical practice, significant contributors of decreased survival included age (years), preoperative creatinine $(\mathrm{mg} / \mathrm{dL})$, severe chronic lung disease, NYHA class III/IV, PVD, and previous cardiac surgery (Table 3).

\section{Sub-group analysis}

We further divided our procedure groups into sub- classifications: mAVR, SAVR, TAVR via trans-femoral access (TAVR-TF) and TAVR via alternative subclavian or transaortic approaches (TAVR-Alt) and re-ran our forward Cox proportional hazard model. This revealed a model that was substantially similar to the first, with age, creatinine, NYHA class, severe chronic lung disease, and re-operative status as being predictive of decreased survival (Table 4). As seen in Figure 2, survival did not differ significantly across the procedure groups when adjusting for major confounders (overall $\mathrm{P}=0.45$ ). 

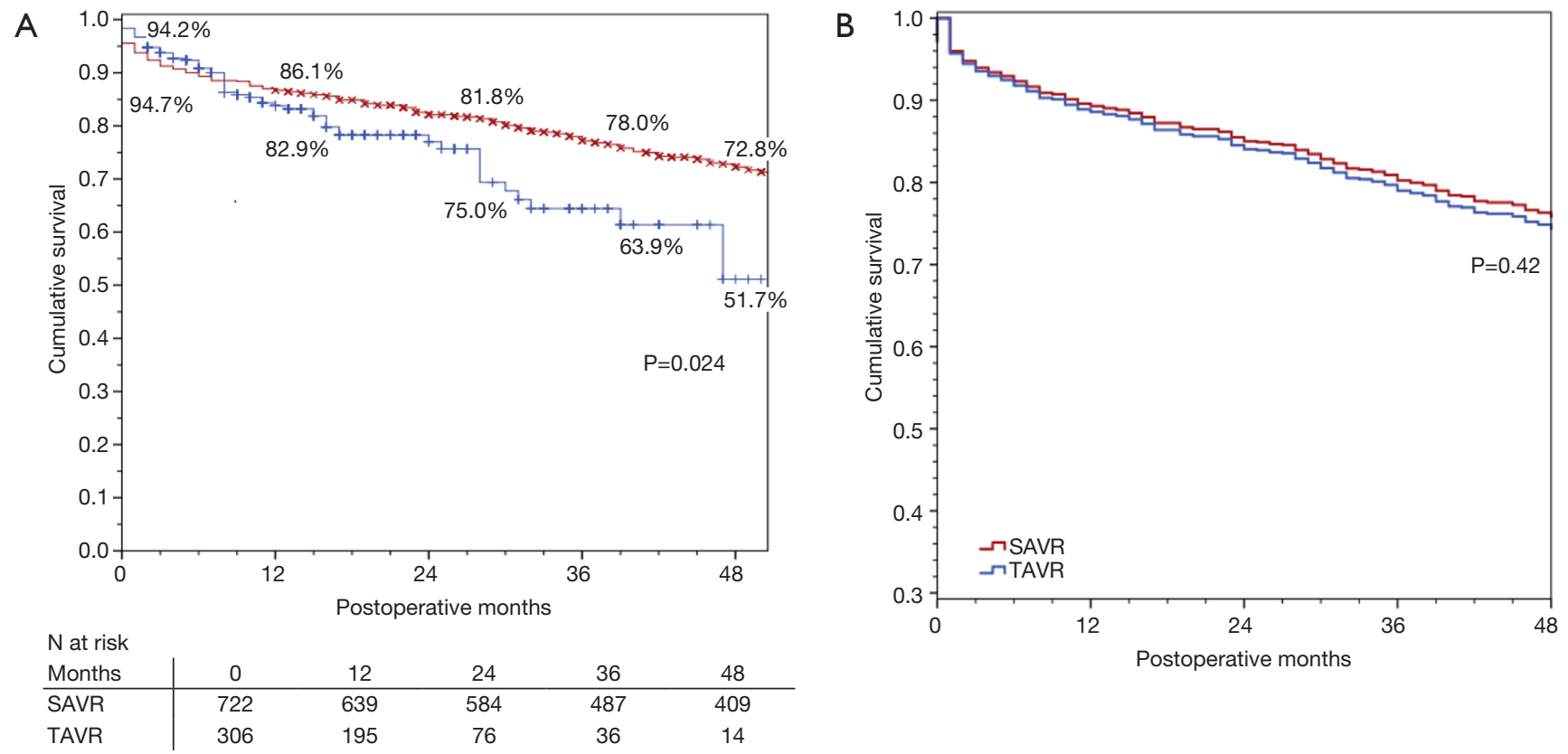

Figure 1 Mid-term survival of surgical-AVR and transcatheter-AVR patients at a high-volume center. (A) Unadjusted Kaplan-Meier and (B) adjusted Cox proportional hazards model.

\begin{tabular}{|c|c|c|c|c|}
\hline \multirow{2}{*}{ Significant variables } & \multirow{2}{*}{$\mathrm{HR}$} & \multicolumn{2}{|c|}{$95 \% \mathrm{Cl}$} & \multirow{2}{*}{$P$ value $(\leq)$} \\
\hline & & Lower & Upper & \\
\hline Age (years) & 1.065 & 1.034 & 1.097 & 0.001 \\
\hline Preoperative creatinine (mg/dL) & 1.182 & 1.095 & 1.279 & 0.001 \\
\hline Chronic lung disease (vs. none) & & & & 0.006 \\
\hline Mild & 1.153 & 0.707 & 1.882 & 0.568 \\
\hline Moderate & 1.319 & 0.816 & 2.134 & 0.259 \\
\hline Severe & 1.857 & 1.101 & 3.130 & 0.020 \\
\hline
\end{tabular}

\section{Discussion}

In our study, we investigated operative and mid-term outcomes of octogenarians undergoing either SAVR or TAVR, further examining the utility of mAVR and alternate access approaches for TAVR. We specifically sought to address the question many clinicians currently face in the current era of TAVR: whether SAVR is still an option among the elderly, especially the octogenarians, who have concurrent multiple co morbidities. This study demonstrated that while TAVR is frequently utilized among octogenarians, especially in a high-risk population, outcomes following SAVR were similar, even after adjusting 


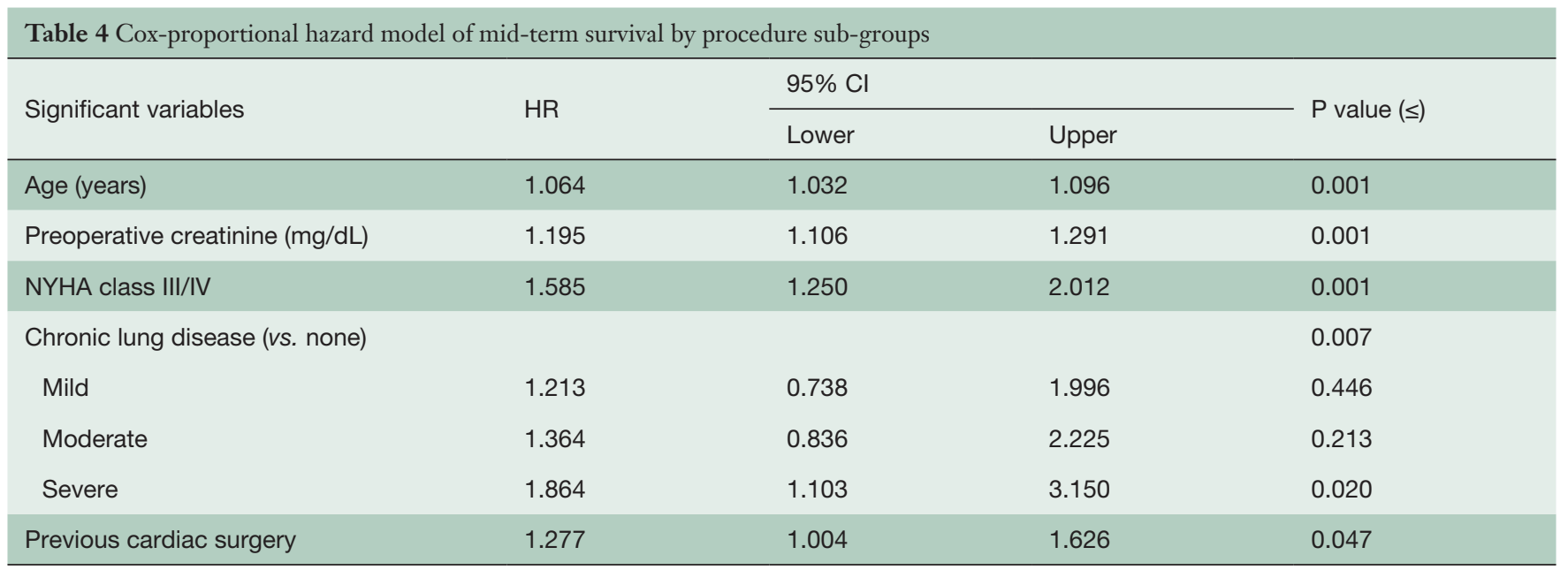



Figure 2 Adjusted mid-term survival by procedure sub-categories: Cox proportional hazards model for mid-term survival, stratified according to the type of procedure [SAVR, minimally invasive SAVR (mAVR), transfemoral TAVR or alternative access TAVR] were not significantly different $(\mathrm{P}=0.45)$.

for various patient-level risk factors. Moreover, both mAVR and SAVR via full sternotomy, appeared to be safe and established treatment options for octogenarians, suggesting that these approaches should continue to be offered in patients with comparable risk factors, especially given the uncertainty of valve performance and duration in TAVR.

Management of aortic disease has evolved considerable in the last decade with emerging transcatheter-based technologies for AVR as an alternative to SAVR via traditional sternotomy. Several recent landmark studies including the PARTNER trials have shown the utility of TAVR in high and intermediate risk patients, with promising short-term results. For instance, the PARTNER IA trial examined the efficacy of TF-TAVI (TAVR) versus standard therapy in inoperable patients with aortic stenosis and found that TAVR patients (which included patients with mean age 83 years) relatively had $20 \%$ less mortality at one year (7). In a similar patient demographic but with higher STS-PROM risk score, PARTNER IB trial showed that TAVI (TAVR) was relatively not inferior at 30 days and 1-year mortality compared to SAVR patients. Notably, rates of major stroke were reportedly similar between the two groups, although vascular complications were significantly more frequent in the SAVR group (10). More recently, the results of PARTNER IIA trial and the recent Surgical Replacement and Transcatheter Aortic Valve Implantation (SURTAVI) trials have further cemented the role of TAVR in intermediate-risk patient groups $(5,11,12)$. Our study, which comprised octogenarians with mostly intermediaterisk scores (means STS-PROM of 6.99) demonstrated similar findings to these trials above during a median follow-up of 35 months. However, we also demonstrated that the type of procedural approach (either full sternotomy SAVR or mAVR, or either TF-TAVR or alternative access) did not significantly impact overall mid-term survival.

Because of the promising results in intermediate-risk patients, some clinicians now have advocated TAVR on all patients over 80 years, despite the lack of long-term data and relative uncertainty over TAVR valve performance over time. However, a normal, healthy 80 -year-old with 
normal body surface area, and no existing co-morbidities undergoing elective AVR will have a low STS-risk score of $1.4 \%$, which will place him as low risk and will not be indicated for TAVR. Using our own extensive 16-year institutional experience with $\mathrm{mAVR}$, we have also demonstrated that the hemi-sternotomy approach is reliable and safe in patients undergoing reoperations and those older than 80 years, with an operative mortality of $2.9 \%$ and longterm survival of $92 \%$ at 10 and 15 years, respectively (9). Likewise, certain individuals with high vascular complication rates and risk factors for PPM, in whom any para-valvular leaks would significantly affect overall survival, may in fact benefit from SAVR as a safe and durable treatment strategy. In our study for instance, we found that that almost a fifth of the patients in the TAVR group had at least trace to moderate residual AR (para-valvular leaks) postoperatively compared to none in the SAVR group. In this context, we believe that SAVR, or preferably mAVR may still have a pivotal role to play in the management of carefully selected patients where avoidance or mitigation of PPM, permanent pacemaker implantation, and other vascular complications would be critical to improve patient prognosis. We also believe that mAVR, instead of SAVR, should be benchmarks against which the performance of emerging TAVR approaches can be compared, especially in octogenarians.

On the other hand, octogenarians, in particular, still remain highly vulnerable to complications given their overall age and frailty, independent of the STS-risk score $(13,14)$. Patients with difficulty ambulating, those with poor nutrition and/or concurrent need for another surgery (due to cancer or orthopedic injuries, for example), and frailty would be ideal candidates for TAVR. Thus, the majority of patients would benefit from TAVR because of faster recovery, improved functional status and quality of life. For instance, a comprehensive systematic review of 60 observational studies by Kim et al. examined functional and quality of life benefits among high-risk patients undergoing TAVR (15). The study found a clinically important decrease from baseline in NYHA class, of up to 2 classes, at 1 and 2 years, as well as improvement in the Short Form-12/36 Health Survey physical component score (change between 4.9 to 26.9 points) by 1 year. Notably, both changes were higher relative to SAVR. Moreover, a few studies assessed other validated measures of functional status such as the Kansas City Cardiomyopathy Questionnaire (KCCQ), and similar clinically important and favorable changes were observed in high-risk and in-operable patients aged 80 years and over (15-18).

The promising results from recent TAVR trials have led to the widespread utilization of TAVR approaches, especially in recipients with a median age of 83 years and a mean age of 81 years, or perhaps older as demonstrated in select patient groups. For instance, recent observational study from three high volume TAVR, mAVR and SAVR centers in the United States showed that between 2011 to 2014, total AVR volume had doubled with notable fivefold increases in TF-TAVR and a 1.5 -fold increase in mAVR. SAVR and TA-TAVR, however, trended down. This trend remained the same, even after risk-stratifying patients according to age $\geq 80$ years, redo AVR, patients receiving dialysis and STS-PROM $>8 \%$ (19). While trends in TAVR utilization are expected to grow with increasing surgeon experiences and innovative transcatheter valvular technologies, unfortunately, contemporary follow-up data on TAVR patients is limited only to 5-7 years in most cases. Moreover, the results of randomized control study in low risk patients (PARTNER 3 trial) will need several more years before we see the results. Some observational studies in lower-risk TAVR populations in Europe have been encouraging to some extent (20-22), but the question regarding valve duration and structural valve deterioration will not be answered anytime soon. Likewise, issues surrounding valve thrombosis, and pacemaker rates are still concerning to many clinicians (23), and yet to be determined in this unique aging population undergoing TAVR. Therefore, treatment decisions should be uniquely tailored according to individual patient profiles and evidence-based assessment of functional and quality of life.

Moreover, there are major costs associated with never TAVR devices, so that widespread adoption will have to be weighed against the associated healthcare costs. For comparison, a study by Simons et al. compared transfemoral TAVR to medical management (calibrated against PARTNER 1 trial), and interestingly found that the incremental cost-effectiveness of TAVR was $\$ 116,500$ per quality-adjusted life-year gained compared to medical management of aortic stenosis, reflecting a substantial and expensive alternative management strategy (24). In a similar study, but one which compared the cost effectiveness of TAVR with a self-expanding prosthesis to SAVR using patient-level data in the CoreValve U.S. High Risk Pivotal Trial, index admission and projected lifetime costs were higher with TAVR than with SAVR (differences $\$ 11,260$ and $\$ 17,849$ per patient, respectively). Specifically, lifetime incremental cost-effectiveness ratios 
were $\$ 55,090$ per quality-adjusted life-year gained with TAVR (25). While these figures are only estimates, the cost disparity is substantial in current era for TAVR in octogenarians, especially when outcomes are comparable with SAVR in the mid-term, and uncertain in the longterm trajectory. In this regard, a multidisciplinary heart team approach is mandatory in order to improve patient selection and healthcare cost-effectiveness. Importantly, as geriatric assessment of frailty has been shown to predict postoperative complications and survival after cardiac surgery, further research is warranted in order to develop robust classification algorithms that accurately predict procedural successes among octogenarians based on longterm survival and validated functional benefits $(14,15,26)$.

While this study has many merits including its large sample size, it has several limitations. First, its observational, single-center, non-randomized design cannot determine causality and may limit generalizability. Moreover, there may be patient selection biases and other inherent confounders that may have affected the study results, although we adjusted for several confounders. Selection criteria for TAVR are different from SAVR, although the former is currently being considered as a potential option for lower-risk patients. We did not have any standard measures to directly account frailty, and our analytic methods are vulnerable to confounding from unmeasured sources of bias. Lastly, there is a substantial difference in the length of follow-up available for patients undergoing TAVR, so it remains to be seen whether survival in these patients continues to be comparable to the results achieved historically. Further prospective studies are warranted that should take into account frailty and valve performance data, as well as procedural costs.

\section{Conclusions}

In octogenarians who underwent AVR, after adjusting for confounders, TAVR, SAVR and mAVR had comparable mortality and mid-term survival. Treatment decisions should be individualized with consensus from a multidisciplinary heart team, taking into account patient co morbidities, frailty and quality of life. We believe certain patient groups will still benefit from SAVR even in this elderly population.

\section{Acknowledgements}

None.

\section{Footnote}

Conflicts of Interest: The authors have no conflicts of interest to declare.

\section{References}

1. Vincent GK, Velkoff VA. The Next Four Decades: The Older Population in the United States: 2010 to 2050. Washington, DC: US Census Bureau, US Government Printing Office, 2010:25-1138.

2. Nkomo VT, Gardin JM, Skelton TN, et al. Burden of valvular heart diseases: a population-based study. Lancet 2006;368:1005-11.

3. Brennan JM, Edwards FH, Zhao Y, et al. Long-term safety and effectiveness of mechanical versus biologic aortic valve prostheses in older patients: results from the Society of Thoracic Surgeons Adult Cardiac Surgery National Database. Circulation 2013;127:1647-55.

4. Brennan JM, Edwards FH, Zhao Y, et al. Long-term survival after aortic valve replacement among high-risk elderly patients in the United States: insights from the Society of Thoracic Surgeons Adult Cardiac Surgery Database, 1991 to 2007. Circulation 2012;126:1621-9.

5. Reardon MJ, Van Mieghem NM, Popma JJ, et al. Surgical or Transcatheter Aortic-Valve Replacement in Intermediate-Risk Patients. N Engl J Med 2017;376:132131.

6. Leon MB, Smith CR, Mack MJ, et al. Transcatheter or Surgical Aortic-Valve Replacement in Intermediate-Risk Patients. N Engl J Med 2016;374:1609-20.

7. Leon MB, Smith CR, Mack M, et al. Transcatheter aorticvalve implantation for aortic stenosis in patients who cannot undergo surgery. N Engl J Med 2010;363:1597-607.

8. Shekar PS. Minimal access aortic valve surgery through an upper hemisternotomy approach. Operative Techniques in Thoracic and Cardiovascular Surgery: A Comparative Atlas 2010;15:321-35

9. Gosev I, Kaneko T, McGurk S, et al. A 16-year experience in minimally invasive aortic valve replacement: context for the changing management of aortic valve disease. Innovations (Phila) 2014;9:104-10; discussion 10.

10. Smith CR, Leon MB, Mack MJ, et al. Transcatheter versus surgical aortic-valve replacement in high-risk patients. N Engl J Med 2011;364:2187-98.

11. Arora S, Ramm CJ, Strassle PD, et al. Review of Major Registries and Clinical Trials of Late Outcomes After Transcatheter Aortic Valve Replacement. Am J Cardiol 
2017;120:331-6.

12. Arora S, Strassle PD, Ramm CJ, et al. Transcatheter Versus Surgical Aortic Valve Replacement in Patients With Lower Surgical Risk Scores: A Systematic Review and Meta-Analysis of Early Outcomes. Heart Lung Circ 2017;26:840-5.

13. Green P, Arnold SV, Cohen DJ, et al. Relation of frailty to outcomes after transcatheter aortic valve replacement (from the PARTNER trial). Am J Cardiol 2015;116:264-9.

14. O'Sullivan CJ, Stortecky S, Buellesfeld L, et al. Preinterventional screening of the TAVI patient: how to choose the suitable patient and the best procedure. Clin Res Cardiol 2014;103:259-74.

15. Kim CA, Rasania SP, Afilalo J, et al. Functional status and quality of life after transcatheter aortic valve replacement: a systematic review. Ann Intern Med 2014;160:243-54.

16. Reynolds MR, Magnuson EA, Lei Y, et al. Health-related quality of life after transcatheter aortic valve replacement in inoperable patients with severe aortic stenosis. Circulation 2011;124:1964-72.

17. Gada H, Kirtane AJ, Wang K, et al. Temporal Trends in Quality of Life Outcomes After Transapical Transcatheter Aortic Valve Replacement: A Placement of AoRTic TraNscathetER Valve (PARTNER) Trial Substudy. Circ Cardiovasc Qual Outcomes 2015;8:338-46.

18. Reynolds MR, Magnuson EA, Wang K, et al. Healthrelated quality of life after transcatheter or surgical aortic valve replacement in high-risk patients with severe aortic stenosis: results from the PARTNER (Placement of AoRTic TraNscathetER Valve) Trial (Cohort A). J Am Coll Cardiol 2012;60:548-58.

19. Nguyen TC, Terwelp MD, Thourani VH, et al. Clinical trends in surgical, minimally invasive and transcatheter aortic valve replacementdagger. Eur J Cardiothorac Surg
2017;51:1086-92.

20. Thyregod HG, Steinbruchel DA, Ihlemann N, et al. No clinical effect of prosthesis-patient mismatch after transcatheter versus surgical aortic valve replacement in intermediate- and low-risk patients with severe aortic valve stenosis at mid-term follow-up: an analysis from the NOTION trial. Eur J Cardiothorac Surg 2016;50:721-8.

21. Thyregod HG, Steinbruchel DA, Ihlemann N, et al. Transcatheter Versus Surgical Aortic Valve Replacement in Patients With Severe Aortic Valve Stenosis: 1-Year Results From the All-Comers NOTION Randomized Clinical Trial. J Am Coll Cardiol 2015;65:2184-94.

22. Thyregod HG, Sondergaard L, Ihlemann N, et al. The Nordic aortic valve intervention (NOTION) trial comparing transcatheter versus surgical valve implantation: study protocol for a randomised controlled trial. Trials 2013;14:11.

23. Webb JG, Mack MJ, White JM, et al. Transcatheter Aortic Valve Implantation Within Degenerated Aortic Surgical Bioprostheses: PARTNER 2 Valve-in-Valve Registry. J Am Coll Cardiol 2017;69:2253-62.

24. Simons CT, Cipriano LE, Shah RU, et al. Transcatheter aortic valve replacement in nonsurgical candidates with severe, symptomatic aortic stenosis: a cost-effectiveness analysis. Circ Cardiovasc Qual Outcomes 2013;6:419-28.

25. Reynolds MR, Lei Y, Wang K, et al. Cost-Effectiveness of Transcatheter Aortic Valve Replacement With a SelfExpanding Prosthesis Versus Surgical Aortic Valve Replacement. J Am Coll Cardiol 2016;67:29-38.

26. Cerrato E, Nombela-Franco L, Nazif TM, et al. Evaluation of current practices in transcatheter aortic valve implantation: The WRITTEN (WoRldwIde TAVI ExperieNce) survey. Int J Cardiol 2017;228:640-7.
Cite this article as: Hirji SA, Ramirez-Del Val F, Kolkailah AA, Ejiofor JI, McGurk S, Chowdhury R, Lee J, Shah PB, Sobieszczyk PS, Aranki SF, Pelletier MP, Shekar PS, Kaneko T. Outcomes of surgical and transcatheter aortic valve replacement in the octogenarians-surgery still the gold standard? Ann Cardiothorac Surg 2017;6(5):453-462. doi: 10.21037/ acs.2017.08.01 